Bull. Korean Math. Soc. 48 (2011), No. 5, pp. 939-949

http://dx.doi.org/10.4134/BKMS.2011.48.5.939

\title{
MATCHING THEOREMS AND SIMULTANEOUS RELATION PROBLEMS
}

\author{
Mircea Balaj and Lucian Coroianu
}

\begin{abstract}
In this paper we give two matching theorems of Ky Fan type concerning open or closed coverings of nonempty convex sets in a topological vector space. One of them will permit us to put in evidence, when $X$ and $Y$ are convex sets in topological vector spaces, a new subclass of $\operatorname{KKM}(X, Y)$ different by any admissible class $\mathfrak{A}_{c}(X, Y)$. For this class of set-valued mappings we establish a KKM-type theorem which will be then used for obtaining existence theorems for the solutions of two types of simultaneous relation problems.
\end{abstract}

\section{Introduction}

In [7] by using his own generalization of the Knaster-Kuratowski-Mazurkievicz theorem (simply, KKM theorem), Ky Fan obtains a matching theorem [7, Theorem 3] for open coverings of convex sets. From this, he also obtains another matching theorem [7, Theorem 4] for closed coverings of convex sets. Shih [24, Theorem 1] establishes the open version of the KKM principle and proves that this is equivalent to Fan's matching theorem for closed covering. Generalizations of these two matching theorems, involving one or more setvalued mappings, have been then obtained in [1], [5], [18], [19], [27].

Two new matching theorems will be obtained in this paper. One of them will permit us to put in evidence, when $X$ and $Y$ are convex sets in topological vector spaces, a new subclass of $\mathbf{K K M}(X, Y)$ different by any admissible class $\mathfrak{A}_{c}(X, Y)$ (see [20]). For this class of set-valued mappings a KKM-type theorem similar to Theorem 7 in [20] will be established.

An abstract variational relation problem was very recently introduced by Luc [15] (see also Khanh and Luc [10], Lin and Ansari [12], Luc et al. [16], Balaj and Lin [2] for further studies) as a model for many problems in optimization, equilibrium theory, variational inclusions or variational inequalities. In [14] Lin and Wang continue the investigation process in this direction studying simultaneous variational relation problems.

Received January 15, 2010.

2010 Mathematics Subject Classification. 47H10, $49 \mathrm{~J} 53$.

Key words and phrases. matching theorem, KKM theorem, $\operatorname{KKM}(X, Y), \mathfrak{A}_{c}(X, Y)$, variational relation. 
Let $X, Y$ and $Z$ be nonempty convex sets in topological vector spaces, $F, G$ : $X \multimap Y, Q: Y \multimap Z$ be set-valued mappings, $R_{1}(y, u)$ be a relation linking elements $y, u \in Y$ and $R_{2}(x, z)$ be a relation linking elements $x \in X$ and $z \in Z$. Motivated by the paper mentioned above, in the last section of the paper we obtain existence theorems for the following two types of simultaneous relation problems:

(I) Find $(\bar{x}, \bar{y}) \in G r(F)$ such that

(a) $R_{1}(\bar{y}, u)$ holds for all $u \in G(\bar{x})$,

(b) for each $x \in X$ there exists $z \in Q(\bar{y})$ for which $R_{2}(x, z)$ holds, and

(II) Find $(\bar{x}, \bar{y}) \in G r(F)$ such that

(a) $R_{1}(\bar{y}, u)$ holds for all $u \in G(\bar{x})$,

(b) for each $x \in X$ and $z \in Q(\bar{y}), R_{2}(x, z)$ holds.

\section{Preliminaries}

In this paper all topological spaces are assumed to be Hausdorff. For a subset $A$ of a topological vector space, the standard notations $\operatorname{co} A$ and $\bar{A}$ designate the convex hull and closure of $A$. To a set-valued mapping $T: X \multimap Y$, we associate the mappings $T^{c}: X \multimap Y, T^{-}: Y \multimap X$, defined by $T^{c}(x)=Y \backslash T(x)$, $T^{-}(y)=\{x \in X: y \in T(x)\}$.

A set-valued mapping $T: X \multimap Y$ is said to be: (i) upper semicontinuous (in short, u.s.c.) (respectively, lower semicontinuous (in short, l.s.c.)) if for every closed subset $B$ of $Y$ the set $\{x \in X: T(x) \cap B \neq \emptyset\}$ (respectively, $\{x \in X$ : $T(x) \subseteq B\}$ ) is closed; (ii) compact if $T(X) \subseteq K$ for some compact set $K$ in $Y$. (iii) closed if its graph (that is, the set $\operatorname{Gr}(T)=\{(x, y) \in X \times Y: y \in T(x)\}$ ) is a closed subset of $X \times Y$.

Recall (see [13] or [26]) that a set-valued mapping $T$ is l.s.c. if and only if for any net $\left\{x_{t}\right\}$ in $X$ converging to $x \in X$ and each $y \in T(x)$ there exist a subnet $\left\{x_{t_{\alpha}}\right\}$ of $\left\{x_{t}\right\}$ and a net $\left\{y_{t_{\alpha}}\right\}$ converging to $y$ with $y_{t_{\alpha}} \in T\left(x_{t_{\alpha}}\right)$ for all $\alpha$.

Assume that $X$ is a convex subset of a vector space and $Y$ is a topological space. If $S, T: X \multimap Y$ are two set-valued mappings such that $T(\operatorname{co} A) \subseteq S(A)$ for each nonempty finite subset $A$ of $X$, then we say that $S$ is a KKM mapping with respect to $T$. A set-valued mapping $T: X \multimap Y$ is said to have the KKM property (see [3]) if for any $S: X \multimap Y$, KKM mapping with respect to $T$, the family $\{\overline{S(x)}: x \in X\}$ has the finite intersection property. Denote $\mathbf{K K M}(X, Y)=\{T: X \multimap Y: T$ has the KKM property $\}$.

For topological spaces $X$ and $Y$, an admissible class $\mathfrak{A}_{c}(X, Y)$ of set-valued mappings $T: X \multimap Y$ (see [20]) is one such that each $T \in \mathfrak{A}_{c}(Y, X)$ is a finite composition of mappings in a class $\mathfrak{A}$ satisfying the following conditions:

(i) $\mathfrak{A}$ contains the class $\mathcal{C}$ of (single-valued) continuous functions;

(ii) each $T \in \mathfrak{A}_{c}$ is u.s.c. with nonempty compact values; and 
(iii) for any polytope $P$ each $T \in \mathfrak{A}_{c}(P, P)$ has a fixed point, where the intermediate spaces are suitable chosen.

It is known that, if $X$ is a convex set in a topological vector space, any admissible class $\mathfrak{A}_{c}(X, Y)$ is a subclass of $\operatorname{KKM}(X, Y)$ (see [20]). Examples of admissible mappings are the Kakutani mappings (with convex values), the acyclic mappings (with acyclic values), the Aronszajn mappings (with $\mathrm{R}_{\delta}$ values), the Fan-Browder mappings ( $\Phi$ mappings) and many others (see $[3,10,12,15])$.

Definition 1. Let $X$ be a convex set in a topological vector space and $Y$ be a convex subset of a vector space. A set-valued mapping $S: X \multimap Y$ is said to be:

(i) quasiconvex (see [17]) if for each convex subset $C$ of $Y, S^{-}(C)$ is convex;

(ii) prequasiconvex if for each convex subset $C$ of $Y, \overline{S^{-}(C)}$ is convex.

Obviously a quasiconvex mapping is prequasiconvex. It can be easily check that a convex mapping (that is, a mapping $S: X \multimap Y$ satisfying $\lambda S\left(x_{1}\right)+(1-$ $\lambda) S\left(x_{2}\right) \subseteq S\left(\lambda x_{1}+(1-\lambda) x_{2}\right)$ for all $x_{1}, x_{2} \in X$ and $\left.\lambda \in[0,1]\right)$ is quasiconvex.

\section{Matching theorems}

Theorem 1. Let $X$ and $Y$ be nonempty convex sets in two topological vector spaces, $\left\{x_{i}: i \in I\right\}$ be a set of points in $X,\left\{Y_{i}: i \in I\right\}$ be an open cover of $Y$ and $T: X \multimap Y$ be a set-valued mapping with nonempty values such that $T^{-}$is prequasiconvex. Suppose that either (i) $T$ is compact, or (ii) the index set $I$ is finite. Then there exists a nonempty finite set of indices $J$ of I such that

$$
T\left(\operatorname{co}\left\{x_{i}: i \in J\right\}\right) \bigcap \bigcap_{i \in J} Y_{i} \neq \emptyset
$$

Proof. Suppose first that $T$ is compact. Then $\widetilde{Y}=\overline{T(X)}$ is a compact subset of $Y$. Moreover, since $T^{-}$is prequasiconvex, $\tilde{Y}$ is convex. Since $\widetilde{Y} \subseteq \bigcup_{i \in I} Y_{i}$, there exists a finite subset $\widetilde{I}$ of $I$ such that $\widetilde{Y} \subseteq \bigcup_{i \in \widetilde{I}} Y_{i}$. Replacing $Y$ and $I$ by $\widetilde{Y}$ and $\widetilde{I}$ respectively, case (i) reduces to (ii).

Hence we will consider that the index set $I$ is finite. We may assume, without loss of generality, that $Y_{i} \neq \emptyset$ and choose $y_{i} \in T\left(x_{i}\right)$ for each $i \in I$. Suppose that the conclusion of the theorem is false. Then for any nonempty subset $J$ of $I$ we have

$$
T\left(\operatorname{co}\left\{x_{i}: i \in J\right\}\right) \subseteq \bigcup_{i \in J} Y_{i}^{c} .
$$

Since $\bigcup_{i \in J} Y_{i}^{c}$ is a closed set, it follows that

$$
\overline{T\left(c o\left\{x_{i}: i \in J\right\}\right)} \subseteq \bigcup_{i \in J} Y_{i}^{c}
$$


Since $T\left(x_{i}\right) \subseteq \overline{T\left(c o\left\{x_{i}: i \in J\right\}\right)}$ for all $i \in J$, and $\overline{T\left(\operatorname{co}\left\{x_{i}: i \in J\right\}\right)}$ is convex, it follows that

$$
\operatorname{co}\left\{y_{i}: i \in J\right\} \subseteq \operatorname{co}\left(\bigcup_{i \in J} T\left(x_{i}\right)\right) \subseteq \overline{T\left(\operatorname{co}\left\{x_{i}: i \in J\right\}\right)},
$$

which implies $c o\left\{y_{i}: i \in J\right\} \subseteq \bigcup_{i \in J} Y_{i}^{c}$.

It now follows, from the well-known Fan-KKM principle [6], that $\bigcap_{i \in I} Y_{i}^{c} \neq$ $\emptyset$, which contradicts the fact that $\left\{Y_{i}: i \in I\right\}$ is a cover of $Y$.

Using as argument the open version of the Fan-KKM principle (see [11] or [25]) one can prove the following closed version of Theorem 1:

Theorem 2. Let $X$ and $Y$ be nonempty convex sets in two topological vector spaces, $I$ be a finite index set, $\left\{x_{i}: i \in I\right\}$ be a set of points in $X$ and $\left\{Y_{i}: i \in I\right\}$ be a closed cover of $Y$. If $T: X \multimap Y$ is a set-valued mapping with nonempty values such that $T^{-}$is quasiconvex, then there exists a nonempty finite set of indices $J$ of $I$ such that

$$
T\left(c o\left\{x_{i}: i \in J\right\}\right) \bigcap \bigcap_{i \in J} Y_{i} \neq \emptyset .
$$

If $X$ and $Y$ are nonempty convex sets in two topological vector spaces we note $\mathfrak{T}(X, Y)=\left\{T: X \multimap Y \mid T\right.$ has nonempty values and $T^{-}$is prequasiconvex $\}$.

We give now two important consequences of Theorem 1 . The first one is Theorem 2.2 in [9].

Theorem 3. If $X$ and $Y$ are nonempty convex sets in two topological vector spaces, then $\mathfrak{T}(X, Y) \subseteq \mathbf{K K M}(X, Y)$.

Proof. Let $S$ be a KKM mapping with respect to $T$ having nonempty closed values. Suppose that there exists a nonempty finite subset $\left\{x_{i}: i \in I\right\}$ of $X$ such that $\bigcap_{i \in I} S\left(x_{i}\right)=\emptyset$. For each $i \in I$ put $Y_{i}=S^{c}\left(x_{i}\right)$. Then, $\left\{Y_{i}: i \in I\right\}$ is an open cover of $Y$. By Theorem 1 there exists a nonempty subset $J$ of $I$ such that $T\left(\operatorname{co}\left\{x_{i}: i \in J\right\}\right) \cap \bigcap_{i \in J} Y_{i} \neq \emptyset$. This relation is equivalent to $T\left(\operatorname{co}\left\{x_{i}: i \in J\right\}\right) \nsubseteq \bigcup_{i \in J} S\left(x_{i}\right)$ which contradicts the fact that $S$ is a KKM mapping with respect to $T$.

Theorem 4. Let $X$ and $Y$ be nonempty convex sets in two topological vector spaces. Let $T, S: X \multimap Y$ be two set-valued mappings with nonempty values such that:

(i) $T \in \mathfrak{T}(X, Y)$ is compact;

(ii) $S$ has closed values;

(iii) $S$ is a KKM mapping with respect to $T$.

Then $\overline{T(X)} \cap \bigcap_{x \in X} S(x) \neq \emptyset$.

Proof. By way of contradiction, suppose that $\overline{T(X)} \cap \bigcap_{x \in X} S(x)=\emptyset$. Then, denoting by $\widetilde{Y}=\overline{T(X)},\left\{S^{c}(x) \cap \tilde{Y}: x \in X\right\}$ is an open cover of the compact 
convex set $\tilde{Y}$. Theorem 1 get a finite subset $\left\{x_{i}: i \in J\right\}$ of $X$ such that $T\left(c o\left\{x_{i}: i \in J\right\}\right) \cap \bigcap_{i \in J} S^{c}\left(x_{i}\right) \neq \emptyset$. But, obviously, this fact contradicts (iii) and thus the proof is complete.

Remark 1. A similar result to Theorem 4 has been obtained by Park in [20, Theorem 7], when $X$ is a convex set in a topological vector space, $Y$ is a topological space and $T \in \mathfrak{A}_{c}(X, Y)$. We show that Theorem 4 and Park's result are independent of each other. More precisely, the examples below, prove that for any admissible class $\mathfrak{A}_{c}(X, Y), \mathfrak{T}(X, Y) \nsubseteq \mathfrak{A}_{c}(X, Y)$, and $\mathfrak{A}_{c}(X, Y) \nsubseteq$ $\mathfrak{T}(X, Y)$.

Let $B$ be the closed unit ball in $\mathbf{R}^{n}(n \geq 2)$. Let $p: \mathbf{R}^{n} \rightarrow B$ be the standard retraction on $B$, that is,

$$
p(x)= \begin{cases}x & \text { if } x \in B \\ \frac{1}{\|x\|} x & \text { if } x \in \mathbf{R}^{n} \backslash B .\end{cases}
$$

Since $p$ is a continuous function it belongs to any admissible class $\mathfrak{A}_{c}\left(\mathbf{R}^{n}, B\right)$. If $C$ is a convex set in $\mathbf{R}^{n}$ with nonempty interior, disjoint from $B$, then $\overline{p(C)}$ is not a convex set, hence $p \notin \mathfrak{T}\left(\mathbf{R}^{n}, B\right)$.

Let $[a, b]$ be a compact real interval and $f, g$ be two real-valued convex functions defined on $[a, b]$, such that $f(x)<g(x)$ for all $x \in[a, b]$ and at least one of them is discontinuous in at least one of the endpoints of the interval. Let $T:[a, b] \multimap \mathbf{R}$ be defined by $T(x)=[f(x), g(x)]$ for each $x \in[a, b]$. By $[17$, p.12, Example 2.2], $T$ is convex, hence $T \in \mathfrak{T}([a, b], \mathbf{R})$. Since its graph is not closed, $T$ is not u.s.c. and consequently $T \notin \mathfrak{A}_{c}([a, b], \mathbf{R})$.

\section{Simultaneous relation problems}

At the beginning of this section we recall some definitions concerning relations and introduce a new one. Let $X$ and $Y$ be convex sets in topological vector spaces. We say that a relation $R(x, y)$ linking elements $x \in X$ and $y \in Y$ is convex (closed, open, respectively) if the set $\{(x, y) \in X \times Y: R(x, y)$ holds $\}$ is convex (closed, open, respectively). The relation $R$ is said to be convex (closed, open, respectively) in the first variable if for each $y \in Y$ the set $\{x \in X: R(x, y)$ holds $\}$ is convex (closed, open, respectively). A relation $R$ on $Y \times Y$ is said to be KKM if for every finite subset $\left\{y_{1}, \ldots, y_{n}\right\}$ of $Y$ and every convex combination $y$ of $y_{1}, \ldots, y_{n}$ one can find some index $i$ such that $R\left(y, y_{i}\right)$ holds.

Having in mind the concepts of pseudomonotone mappings and weak pseudomonotone mappings (see [8]) we give the following:

Definition 2. Let $X, Y$ and $Z$ be nonempty sets, $G: X \multimap Y, Q: Y \multimap Z$ be set-valued mappings, $R_{1}(y, u)$ be a relation linking elements $y, u \in Y$ and $R_{2}(x, z)$ be a relation linking elements $x \in X$ and $z \in Z$. We say that: 
(i) $R_{2}$ is $R_{1}$ weak pseudomonotone with respect to $(Q, G)$ if the following implication holds: $x \in X, y \in Y, R_{1}(y, u)$ holds for all $u \in G(x) \Longrightarrow$ $R_{2}(x, z)$ holds for some $z \in Q(y)$;

(ii) $R_{2}$ is $R_{1}$ pseudomonotone with respect to $(Q, G)$ if the following implication holds: $x \in X, y \in Y, R_{1}(y, u)$ holds for all $u \in G(x) \Longrightarrow R_{2}(x, z)$ holds for all $z \in Q(y)$.

Theorem 5. Let $X, Y$ and $Z$ be nonempty convex sets in topological vector spaces, $X$ and $Y$ being compact. Let $F, G: X \multimap Y, Q: Y \multimap Z$ be set-valued mappings, $R_{1}(y, u)$ a relation linking elements $y, u \in Y$ and $R_{2}(x, z)$ a relation linking elements $x \in X$ and $z \in Z$. Suppose that:

(i) $G \subseteq F, F$ is u.s.c. and convex with compact convex values, $G$ is l.s.c. and concave with nonempty values;

(ii) $Q$ is u.s.c.;

(iii) the relation $R_{1}$ is KKM, closed and convex;

(iv) the relation $R_{2}$ is closed in the second variable and $R_{2}^{c}$ is convex in the first variable, where $R_{2}^{c}$ denotes the complement of the relation $R_{2}$ (that is, $R_{2}^{c}(x, z)$ holds if and only if $R_{2}(x, z)$ does not hold);

(v) $R_{2}$ is $R_{1}$ weak pseudomonotone with respect to $(Q, G)$.

Then there exists $(\bar{x}, \bar{y}) \in G r(F)$ such that

(a) $R_{1}(\bar{y}, u)$ holds for all $u \in G(\bar{x})$,

(b) for each $x \in X$ there exists $z \in Q(\bar{y})$ for which $R_{2}(x, z)$ holds.

Proof. The proof is divided into three steps. by

Step 1. We shall prove that the multivalued mapping $T: X \multimap Y$ defined belongs to $\mathfrak{T}(X, Y)$.

$$
T(x)=\left\{y \in F(x): R_{1}(y, u) \text { holds for all } u \in G(x)\right\}
$$

For each $x \in X, T(x)$ is nonempty. Indeed, let $B_{x}: G(x) \multimap F(x)$ be defined by $B_{x}(u)=\left\{y \in F(x): R_{1}(y, u)\right.$ holds $\}$ for all $u \in G(x)$. Since $R_{1}$ is closed and $F(x)$ is compact, the mapping $B_{x}$ has compact values. Let $A$ be a nonempty finite subset of $G(x)$ and $y \in \operatorname{coA}$. Then $y \in F(x)$ and, since the relation $R_{1}$ is KKM, $y \in B_{x}(A)$. Hence $B_{x}$ is a KKM mapping and by the Fan-KKM theorem there exists $y_{x} \in F(x)$ such that $y_{x} \in \bigcap_{u \in G(x)} B_{x}(u)$. Then $R_{1}\left(y_{x}, u\right)$ holds for all $u \in G(x)$, hence $y_{x} \in T(x)$ for each $x \in X$.

Let $C$ be a convex subset of $X$. We claim that $T(C)$ is convex. Let $y_{1}, y_{2} \in$ $T(C)$ and $y=\lambda y_{1}+(1-\lambda) y_{2}$ a convex combination of $y_{1}, y_{2}(\lambda \in[0,1])$. For $i=1,2$ there exists $x_{i} \in C$ such that $y_{i} \in F\left(x_{i}\right)$ and $R_{1}\left(y_{i}, u\right)$ holds for all $u \in$ $G\left(x_{i}\right)$. Let $x=\lambda x_{1}+(1-\lambda) x_{2}$. Since $C$ is convex, $x \in C$. Since the mapping $F$ is convex, $y \in \lambda F\left(x_{1}\right)+(1-\lambda) F\left(x_{2}\right) \subseteq F(x)$. For each $u \in G(x)$, there exist $u_{1} \in G\left(x_{1}\right), u_{2} \in G\left(x_{2}\right)$ such that $u=\lambda u_{1}+(1-\lambda) u_{2}$. Since $R_{1}\left(y_{1}, u_{1}\right)$, $R_{1}\left(y_{2}, u_{2}\right)$ hold and $R_{1}$ is convex, $R_{1}(y, u)$ is satisfied, hence $y \in T(x) \subseteq T(C)$.

Step 2. We show that the range of $T$ is compact. Since $T(X)$ is a subset of the compact $Y$ it suffices to prove that $T(X)$ is closed. Let $y \in \overline{T(X)}$ and 
$\left\{y_{t}\right\}_{t \in \Delta}$ a net in $T(X)$ converging to $y$. For each $t \in \Delta$ there exists $x_{t} \in X$ such that $y_{t} \in F\left(x_{t}\right)$ and $R_{1}\left(y_{t}, u\right)$ holds for all $u \in G\left(x_{t}\right)$. Taking into account the compactness of $X$, we may suppose that the net $\left\{x_{t}\right\}_{t \in \Delta}$ converges to a point $x \in X$. Since $F$ is closed, $y \in F(x)$. Let $u \in G(x)$ be arbitrarily chosen. Since $G$ is l.s.c., there exist a subnet $\left\{x_{t_{\alpha}}\right\}$ of $\left\{x_{t}\right\}$ and a net $\left\{u_{t_{\alpha}}\right\}$ converging to $u$ with $u_{t_{\alpha}} \in G\left(x_{t_{\alpha}}\right)$ for all $\alpha$. Since $R_{1}\left(y_{t_{\alpha}}, u_{t_{\alpha}}\right)$ holds for all $\alpha$ and $R_{1}$ is closed, $R_{1}(y, u)$ holds too. So, $y \in T(x)$, hence $T(X)$ is closed.

Step 3. Consider the set valued-mappings $P: X \multimap Z$ and $S: X \multimap Y$, given by

$P(x)=\left\{z \in Z: R_{2}(x, z)\right.$ holds $\}, S(x)=\{y \in Y: P(x) \cap Q(y) \neq \emptyset\}$ for all $x \in X$,

By (iv), $P(x)$ is a closed set in $Z$ for each $x \in X$. Since $Q$ is u.s.c., $S$ has closed values.

We claim that $S$ is a KKM mapping with respect to $T$. If not, there exists a finite subset $A=\left\{x_{1}, \ldots, x_{n}\right\}$ of $X, x \in c o A$ and $y \in T(x) \backslash S(A)$. Then,

$$
y \in F(x) \text { and } R_{1}(y, u) \text { holds for all } u \in G(x)
$$

and

$$
\text { for each } x_{i} \in A \text { and } z \in Q(y), R_{2}\left(x_{i}, z\right) \text { does not hold. }
$$

Since $R_{2}^{c}$ is convex in the first variable, by $(2), R_{2}(x, z)$ does not hold for all $z \in Q(y)$. On the other hand, by (1) and (v), there exists $z \in Q(y)$ such that $R_{2}(x, z)$ holds; a contradiction. Consequently $S$ is a KKM mapping with respect to $T$.

By Theorem 4, there exists $\bar{y} \in T(X) \cap \bigcap_{x \in X} S(x)$. By $\bar{y} \in T(X)$, there exists $\bar{x} \in X$ such that $\bar{y} \in F(\bar{x})$ and $R_{1}(\bar{y}, u)$ holds for all $u \in G(\bar{x})$. By $\bar{y} \in \bigcap_{x \in X} S(x)$, for each $x \in X$ there exists $z \in Q(\bar{y})$ for which $R_{2}(x, z)$ holds.

Theorem 6. Let $X, Y, Z, Q, G, R_{1}$ and $R_{2}$ be as in Theorem 5. Assume that conditions (i) and (iii) in Theorem 5 and the following conditions are satisfied:

(iii) $Q$ is l.s.c. with convex values;

(iv) the relation $R_{2}$ is open in the second variable and $R_{2}^{c}$ is convex;

(v) $R_{2}$ is $R_{1}$ pseudomonotone with respect to $(Q, G)$.

Then there exists $(\bar{x}, \bar{y}) \in G r(F)$ such that

(a) $R_{1}(\bar{y}, u)$ holds for all $u \in G(\bar{x})$,

(b) $R_{2}(x, z)$ holds for all $x \in X$ and $z \in Q(\bar{y})$.

Proof. Let the set-valued mapping $T$ be defined as in the proof of Therem 5 . We have seen that under the given conditions $T \in \mathfrak{T}(X, Y)$ and $T(X)$ is compact. Let $S: X \multimap Y$ be defined by $S(x)=\left\{y \in Y: R_{2}(x, z)\right.$ holds for all $\left.z \in Q(y)\right\}$. It readily follows that $S$ has closed values (by (ii) and (iv)) and that $S$ is a KKM mapping with respect to $T$ (by (iv) and (v)). 
By Theorem 4, there exists $\bar{y} \in T(X) \bigcap \bigcap_{x \in X} S(x)$. By $\bar{y} \in T(X)$, there exists $\bar{x} \in X$ such that $\bar{y} \in F(\bar{x})$ and $R_{1}(\bar{y}, u)$ holds for all $u \in G(\bar{x})$. By $\bar{y} \in \bigcap_{x \in X} S(x)$ for each $x \in X$ and $z \in Q(\bar{y}), R_{2}(x, z)$ holds.

In a relation problem the given relations are frequently expressed by equalities and inequalities of real functions or by inclusions or intersections of setvalued mappings. In this cases conditions (iii) and (iv) from Theorems 5 and 6 are provided by suitable conditions on the involving mappings. For illustration, we fix now our attention on the relations that appear in vector equilibrium problems. To this aim let us consider two convex sets $A$ and $B$ in topological vector spaces, a topological vector space $V$ and two set-valued mappings $H: A \times B \multimap V, C: A \multimap V$. Define the following relations on $A \times B$ :

(i) $R(a, b)$ holds if and only if $H(a, b) \subseteq C(a)$;

(ii) $R^{\prime}(a, b)$ holds if and only if $H(a, b) \cap C(a) \neq \emptyset$.

There is an extended literature regarding sufficient conditions in order that relations $R, R^{\prime}$ (both or one of them) should be KKM, closed or convex. In the next proposition we review some of them. The proofs are standard and for this reason we give the proof for the first one, only.

Proposition 7. (i) The relation $R$ is:

$\left(\mathrm{i}_{1}\right) K K M$ whenever $A=B, H$ is convex in the second variable, $C^{c}$ is convex-valued and $H(a, a) \subseteq C(a)$ for all $a \in A$;

$\left(\mathrm{i}_{2}\right)$ closed if $H$ is l.s.c. and $C$ is closed;

$\left(\mathrm{i}_{3}\right)$ convex if $H$ is concave and $C$ is convex;

$\left(\mathrm{i}_{4}\right)$ closed in the second variable if $H$ is l.s.c. in the second variable and $C$ is a closed-valued mapping;

$\left(\mathrm{i}_{5}\right)$ open in the second variable if $H$ is a compact-valued mapping, u.s.c. in the second variable and $C$ is an open-valued mapping.

(ii) The relation $R^{c}$ is convex if the set-valued mappings $H$ and $C^{c}$ are convex.

Proof. (i $\left.\mathrm{i}_{1}\right)$ Suppose that there exists a finite subset $\left\{a_{1}, \ldots, a_{n}\right\}$ of $A$ and a convex combination of them, $a=\sum_{i=1}^{n} \lambda_{i} a_{i}$ such that $R\left(a, a_{i}\right)$ does not hold for all index $i$. Then for each $i$ there is $v_{i} \in H\left(a, a_{i}\right) \cap C^{c}(a)$. By the given hypotheses it follows that

$$
\sum_{i=1}^{n} \lambda_{i} v_{i} \in\left(\sum_{i=1}^{n} \lambda_{i} H\left(a, a_{i}\right)\right) \cap C^{c}(a) \subseteq H(a, a) \cap C^{c}(a) \text {; a contradiction. }
$$

$\left(\mathrm{i}_{2}\right)$ Let $\left\{\left(a_{t}, b_{t}\right)\right\}$ be a net converging to $(a, b)$ such that $R\left(a_{t}, b_{t}\right)$ holds for all $t$. If $v \in H(a, b)$, since $H$ is l.s.c. there exist a subnet $\left\{\left(a_{t_{\alpha}}, b_{t_{\alpha}}\right)\right\}$ of $\left\{\left(a_{t}, b_{t}\right)\right\}$ and a net $\left\{v_{t_{\alpha}}\right\}$ converging to $v$, with $v_{t_{\alpha}} \in H\left(a_{t_{\alpha}}, b_{t_{\alpha}}\right) \subseteq C\left(a_{t_{\alpha}}\right)$. Since $C$ is closed, $v \in C(a)$ hence $R(a, b)$ holds. 
$\left(\mathrm{i}_{3}\right)$ Assume that $R\left(a_{1}, b_{1}\right)$ and $R\left(a_{2}, b_{2}\right)$ are satisfied. Then for any $\lambda \in[0,1]$ we have

$$
\begin{aligned}
H\left(\lambda a_{1}+(1-\lambda) a_{2}, \lambda b_{1}+(1-\lambda) b_{2}\right) & \subseteq \lambda H\left(a_{1}, b_{1}\right)+(1-\lambda) H\left(a_{2}, b_{2}\right) \\
& \subseteq \lambda C\left(a_{1}\right)+(1-\lambda) C\left(a_{2}\right) \\
& \subseteq C\left(\lambda a_{1}+(1-\lambda) a_{2}\right) .
\end{aligned}
$$

Hence $R\left(\lambda a_{1}+(1-\lambda) a_{2}, \lambda b_{1}+(1-\lambda) b_{2}\right)$ holds.

$\left(i_{4}\right)$ Let $a \in A$ and let $\left\{b_{t}\right\}$ be a net converging to a point $b$ such that $R\left(a, b_{t}\right)$ holds for all $t$. If $v$ is an arbitrary point in $H(a, b)$, since $H(a, \cdot)$ is l.s.c., there are a subnet $\left\{b_{t_{\alpha}}\right\}$ of $\left\{b_{t}\right\}$ and a net $\left\{v_{t_{\alpha}}\right\}$ converging to $v$ with $v_{t_{\alpha}} \in H\left(a, b_{t_{\alpha}}\right) \subseteq C(a)$. Since $C(a)$ is a closed set, $v \in C(a)$. Thus $H(a, b) \subseteq C(a)$, hence $R(a, b)$ holds.

(i5) We have to show that for $a \in A$ arbitrarily fixed the set $M=\{b \in B$ : $R(a, b)$ does not hold $\}$ is closed. Let $b \in \bar{M}$ and $\left\{b_{t}\right\}$ be a net in $M$ converging to $b$. Then for each $t$ there exists $v_{t} \in H\left(a, b_{t}\right) \cap C^{c}(a)$. Since $H(a, \cdot)$ is u.s.c. with compact values, by Proposition 2.1 in [4], there exist $v \in H(a, b)$ and a subnet $\left\{v_{t_{\alpha}}\right\}$ of $\left\{v_{t}\right\}$ converging to $v$. Since $C^{c}(a)$ is a closed set, $v \in C^{c}(a)$. Thus $b \in M$.

(ii) Let $\left(a_{1}, b_{1}\right),\left(a_{2}, b_{2}\right) \in A \times B$ such that $R^{c}\left(a_{1}, b_{1}\right)$ and $R^{c}\left(a_{2}, b_{2}\right)$ hold. Then, for $i=1,2$ there is $v_{i} \in H\left(a_{i}, b_{i}\right) \cap C^{c}\left(a_{i}\right)$. For any $\lambda \in[0,1]$ we have

$$
\begin{aligned}
\lambda v_{1}+(1-\lambda) v_{2} & \in\left(\lambda H\left(a_{1}, b_{1}\right)+(1-\lambda) H\left(a_{2}, b_{2}\right)\right) \cap\left(\lambda C^{c}\left(a_{1}\right)+(1-\lambda) C^{c}\left(a_{2}\right)\right) \\
& \subseteq H\left(\lambda a_{1}+(1-\lambda) a_{2}, \lambda b_{1}+(1-\lambda) b_{2}\right) \cap C^{c}\left(\lambda a_{1}+(1-\lambda) a_{2}\right) .
\end{aligned}
$$

Hence $R^{c}$ is convex in the first variable.

Proposition 8. (i) The relation $R^{\prime}$ is:

(i $\left.i_{1}\right) K K M$ whenever $A=B, H$ is concave in the second variable and for each $a \in A, C^{c}(a)$ is a convex cone and $H(a, a) \cap C(a) \neq \emptyset$;

(i $\left.i_{2}\right)$ closed if $H$ is u.s.c. with compact values and $C$ is closed;

$\left(\mathrm{i}_{3}\right)$ convex if $H$ and $C$ are convex;

(i $\left.i_{4}\right)$ closed in the second variable if $H$ is a compact-valued mapping, u.s.c. in the second variable and $C$ is a closed-valued mapping;

$\left(\mathrm{i}_{5}\right)$ open in the second variable if $H$ is l.s.c. in the second variable and $C$ is open-valued.

(ii) ${R^{\prime}}^{c}$ is convex if $H$ is concave and $C^{c}$ is convex.

\section{References}

[1] M. Balaj, Admissible maps, intersection results, coincidence theorems, Comment. Math. Univ. Carolin. 42 (2001), no. 4, 753-762.

[2] M. Balaj and L. J. Lin, Generalized variational relation problems with applications, J. Optim. Theory Appl. 148 (2011), 1-13.

[3] T. H. Chang and C. L. Yen, KKM property and fixed point theorems, J. Math. Anal. Appl. 203 (1996), no. 1, 224-235. 
[4] C. R. Chen, S. J. Li, and K. L. Teo, Solution semicontinuity of parametric generalized vector equilibrium problems, J. Global Optim. 45 (2009), no. 2, 309-318.

[5] X. P. Ding, Xie Ping, and K. K. Tan, Matching theorems, fixed point theorems and minimax inequalities without convexity, J. Austral. Math. Soc. Ser. A 49 (1990), no. 1, $111-128$.

[6] K. Fan, A generalization of Tychonoff's fixed point theorem, Math. Ann. 142 (1961), 305-310.

7] _ Some properties of convex sets related to fixed point theorems, Math. Ann. 266 (1984), no. 4, 519-537.

[8] J. Y. Fu and A. H. Wan, Generalized vector equilibrium problems with set-valued mappings, Math. Methods Oper. Res. 56 (2002), no. 2, 259-268.

[9] J. C. Jeng, Y. Y. Huang, and H. L. Zhang, Characterization of maps having the KKM property, Soochow J. Math. 28 (2002), no. 3, 329-338.

[10] P. Q. Khanh and D. T. Luc, Stability of solutions in parametric variational relation problems, Set-Valued Anal. 16 (2008), no. 7-8, 1015-1035.

[11] W. K. Kim, Some applications of the Kakutani fixed point theorem, J. Math. Anal. Appl. 121 (1987), no. 1, 119-122.

[12] L. J. Lin and Q. H. Ansari, Systems of quasi-variational relations with applications, Nonlinear Anal. 72 (2010), no. 3-4, 1210-1220.

[13] L. J. Lin and W. S. Du, Systems of equilibrium problems with applications to new variants of Ekeland's variational principle, fixed point theorems and parametric optimization problems, J. Global Optim. 40 (2008), no. 4, 663-677.

[14] L. J. Lin and S. Y. Wang, Simultaneous variational relation problems and related applications, Comput. Math. Appl. 58 (2009), no. 9, 1711-1721.

[15] D. T. Luc, An abstract problem in variational analysis, J. Optim. Theory Appl. 138 (2008), no. 1, 65-76.

[16] D. T. Luc, E. Sarabi, and A. Soubeyran, Existence of solutions in variational relation problems without convexity, J. Math. Anal. Appl. 364 (2010), no. 2, 544-555.

[17] K. Nikodem, K-Convex and K-Concave Set-Valued Functions, Politechnika, Lodzks, 1989.

[18] S. Park, Generalizations of Ky Fan's matching theorems and their applications, J. Math. Anal. Appl. 141 (1989), no. 1, 164-176.

[19] Some coincidence theorems on acyclic multifunctions and applications to KKM theory, in "Fixed Point Theory and Applications" (K.-K. Tan, Ed.), pp. 248-277, World Scientific, River Edge, New Jersey, 1992.

[20] - Foundations of the KKM theory via coincidences of composites of upper semicontinuous maps, J. Korean Math. Soc. 31 (1994), no. 3, 493-519.

[21] _ A unified fixed point theory of multimaps on topological vector spaces, J. Korean Math. Soc. 35 (1998), no. 4, 803-829. Corrections, ibid. 36 (1999), 829-832.

[22] _ Fixed points of better admissible maps on generalized convex spaces, J. Korean Math. Soc. 37 (2000), no. 6, 885-899.

[23] S. Park and H. Kim, Admissible classes of multifunctions on generalized convex spaces, Proc. Coll. Natur. Sci. Seoul National University 18 (1993), 1-21.

[24] M. H. Shih, Covering properties of convex sets, Bull. London Math. Soc. 18 (1986), no. $1,57-59$.

[25] M. H. Shih and K. K. Tan, Covering theorems of convex sets related to fixed-point theorems, in "Nonlinear and Convex Analysis" (B. L. Lin, S. Simons, eds.), Marcel Dekker, New York, 1987.

[26] N. X. Tan, Quasivariational inequalities in topological linear locally convex Hausdorff spaces, Math. Nachr. 122 (1985), 231-245.

[27] S. S. Zhang and Z. Zhang, On a class of new KKM theorem with applications, Appl. Math. Mech. 17 (1996), no. 9. 773-780. 
Mircea BALAJ

Department of Mathematics

UNIVERSITY OF ORADEA

ROMANIA

E-mail address: mbalaj@uoradea.ro

LUCIAN COROIANU

Department of Mathematics

UNIVERSITY OF ORADEA

ROMANIA

E-mail address: 1coroianu@uoradea.ro 